\title{
BDD Representation for Incompletely Specified Multiple-Output Logic Functions and Its Applications to Functional Decomposition
}

\author{
Tsutomu Sasao and Munehiro Matsuura \\ Department of Computer Science and Electronics, \\ Kyushu Institute of Technology, \\ lizuka 820-8502, Japan
}

\begin{abstract}
A multiple-output function can be represented by a binary decision diagram for characteristic function (BDD_for_CF). This paper presents a new method to represent multiple-output incompletely specified functions using BDD_for_CF. An algorithm to reduce the widths of BDD_for_CFs is presented. This method is useful for decomposition of incompletely specified multipleoutput functions. Experimental results for radix converters, adders and a multiplier show that this method is useful for the synthesis of LUT cascades. This data structure is also useful to threevalued logic simulation.
\end{abstract}

\section{Categories and Subject Descriptors}

B.6.3 [Logic Design]: Design Aids

\section{General Terms}

Algorithms, Design, Experimentation, Performance, Theory

\section{Keywords}

Incompletely Specified Function, BDD, Characteristic function, Cascade, Code converter

\section{INTRODUCTION}

Construction of a Binary Decision Diagram (BDD) for an incompletely specified Boolean function arises in several applications in the CAD domain: verification, logic synthesis, and software synthesis. Three methods are known to represent an incompletely specified logic function by binary decision diagrams (BDDs) [9]:

1. A ternary function that takes 0,1 and don't care [9].

2. A pair of BDDs to represent three values [4].

3. An auxiliary variable that represents don't cares [9, 3].

Most works are related to the minimization of total number of nodes in BDDs $[3,6,17,18]$. However, these methods are unsuitable for functional decompositions of multiple-output functions. In a functional decomposition, the minimization of width

Permission to make digital or hard copies of all or part of this work for personal or classroom use is granted without fee provided that copies are not made or distributed for profit or commercial advantage and that copies bear this notice and the full citation on the first page. To copy otherwise, to republish, to post on servers or to redistribute to lists, requires prior specific permission and/or a fee.

DAC 2005, June 13-17, 2005, Anaheim, California, USA.

Copyright 2005 ACM 1-59593-058-2/05/0006 ...\$5.00. of a BDD is more important than the minimization of total number of nodes. To find an efficient decomposition of a multipleoutput logic function, we can use a multi-terminal binary decision diagram (MTBDD), or a BDD that represents the characteristic function of the multiple-output function (BDD_for_CF) [14]. BDD_for_CFs usually require fewer nodes than corresponding MTBDDs, and the widths of the BDD_for_CFs tend to be smaller than that of the corresponding MTBDDs.

In this paper, we show a new method to represent an incompletely specified multiple-output function. It uses a BDD_for_CF, and is suitable for functional decomposition. We also show a method to reduce the width of the BDD_for_CF. Experimental results using radix converters, adders, and a multiplier show the effectiveness of the approach.

\section{DEFINITIONS}

DEFINITION 2.1. $x$ is a support variable of $f$ if $f$ depends on $x$. A function $f:\{0,1\}^{n} \rightarrow\{0,1, d\}$ is an incompletely specified function, where $d$ denotes the don't care. Let $f_{-}, f_{-1}$, and $f_{-d}$ be the functions represented by sets $f^{-1}(0), f^{-1}(1)$, and $f^{-1}(d)$, respectively. Note that $f_{-} \vee f_{-1} \vee f_{-d}=1, f_{-} \cdot f_{-1}=0$, $f_{-1} \cdot f_{-d}=0$, and $f_{-} 0 \cdot f_{-d}=0$.

Definition 2.2. [2] Let $F=\left(f_{1}(X), f_{2}(X), \ldots, f_{m}(X)\right)$ be a multiple-output function, and let $X=\left(x_{1}, x_{2}, \ldots, x_{n}\right)$ be the input variables. The characteristic function of the completely specified multiple-output function $F$ is

$$
\chi(X, Y)=\bigwedge_{i=1}^{m}\left(y_{i} \equiv f_{i}(X)\right),
$$

where $y_{i}$ is the variable representing the output $f_{i}$, and $i \in\{0,1$, $\ldots, m\}$.

The characteristic function of a completely specified multipleoutput function denotes the set of the valid input-output combinations. Let $f_{i_{-} 0}(X)=\bar{f}_{i}(X)$ and $f_{i_{-} 1}(X)=f_{i}(X)$, then the characteristic function $\chi$ is represented as follows:

$$
\chi(X, Y)=\bigwedge_{i=1}^{m}\left(\bar{y}_{i} \cdot f_{i_{-} 0}(X) \vee y_{i} \cdot f_{i_{-} 1}(X)\right) .
$$

In an incompletely specified function, when the function value $f_{i}$ is don't care, the value of the function can be either 0 or 1 . Therefore, for such inputs, the characteristic function is independent of the values of output variables $y_{i}$. Let $f_{i \_d}$ denote the don't care set, then we have

$$
\begin{gathered}
\bar{y}_{i}\left(f_{i_{-} 0}(X) \vee f_{i_{-} d}(X)\right) \vee y_{i}\left(f_{i_{1} 1}(X) \vee f_{i_{-} d}(X)\right) \\
\quad=\bar{y}_{i} f_{i_{-} 0}(X) \vee y_{i} f_{i_{-} 1}(X) \vee f_{i_{-} d}(X)
\end{gathered}
$$


Table 2.1: Truth table of an incompletely specified function.

\begin{tabular}{|cccc|cc||cccc|cc|}
\hline$x_{1}$ & $x_{2}$ & $x_{3}$ & $x_{4}$ & $f_{1}$ & $f_{2}$ & $x_{1}$ & $x_{2}$ & $x_{3}$ & $x_{4}$ & $f_{1}$ & $f_{2}$ \\
\hline 0 & 0 & 0 & 0 & $d$ & 1 & 1 & 0 & 0 & 0 & 0 & 1 \\
0 & 0 & 0 & 1 & $d$ & 1 & 1 & 0 & 0 & 1 & 0 & 1 \\
0 & 0 & 1 & 0 & 0 & 0 & 1 & 0 & 1 & 0 & 1 & 0 \\
0 & 0 & 1 & 1 & 0 & 0 & 1 & 0 & 1 & 1 & 1 & 0 \\
0 & 1 & 0 & 0 & $d$ & $d$ & 1 & 1 & 0 & 0 & 1 & $d$ \\
0 & 1 & 0 & 1 & $d$ & $d$ & 1 & 1 & 0 & 1 & 1 & $d$ \\
0 & 1 & 1 & 0 & 1 & 0 & 1 & 1 & 1 & 0 & $d$ & 0 \\
0 & 1 & 1 & 1 & 1 & 1 & 1 & 1 & 1 & 1 & $d$ & 1 \\
\hline
\end{tabular}

Thus, we have the following:

DEFINITION 2.3. The characteristic function $\chi$ of an incompletely specified multiple-output function $F=\left(f_{1}(X), f_{2}(X), \ldots, f_{m}(X)\right)$ is

$$
\chi(X, Y)=\bigwedge_{i=1}^{m}\left\{\bar{y}_{i_{i}} f_{i_{-} 0}(X) \vee y_{i} f_{i_{-} 1}(X) \vee f_{i_{-} d}(X)\right\}
$$

EXAMPLE 2.1. Consider the incompletely specified function shown in Table 2.1. Since,

$$
\begin{aligned}
f_{1 \_0} & =\bar{x}_{1} \bar{x}_{2} x_{3} \vee x_{1} \bar{x}_{2} \bar{x}_{3} \\
f_{1 \_1} & =\bar{x}_{1} x_{2} x_{3} \vee x_{1} \bar{x}_{2} x_{3} \vee x_{1} x_{2} \bar{x}_{3} \\
f_{1 \_d} & =\bar{x}_{1} \bar{x}_{3} \vee x_{1} x_{2} x_{3} \\
f_{2 \_0} & =\bar{x}_{1} \bar{x}_{2} x_{3} \vee x_{1} \bar{x}_{2} x_{3} \vee x_{2} x_{3} \bar{x}_{4} \\
f_{2 \_1} & =\bar{x}_{2} \bar{x}_{3} \vee x_{2} x_{3} x_{4} \\
f_{2 \_} & =x_{2} \bar{x}_{3},
\end{aligned}
$$

the characteristic function is

$$
\begin{aligned}
\chi= & \left\{\bar{y}_{1}\left(\bar{x}_{1} \bar{x}_{2} x_{3} \vee x_{1} \bar{x}_{2} \bar{x}_{3}\right) \vee\right. \\
& \left.y_{1}\left(\bar{x}_{1} x_{2} x_{3} \vee x_{1} \bar{x}_{2} x_{3} \vee x_{1} x_{2} \bar{x}_{3}\right) \vee\left(\bar{x}_{1} \bar{x}_{3} \vee x_{1} x_{2} x_{3}\right)\right\} . \\
& \left\{\bar{y}_{2}\left(\bar{x}_{1} \bar{x}_{2} x_{3} \vee x_{1} \bar{x}_{2} x_{3} \vee x_{2} x_{3} \bar{x}_{4}\right) \vee\right. \\
& \left.y_{2}\left(\bar{x}_{2} \bar{x}_{3} \vee x_{2} x_{3} x_{4}\right) \vee\left(x_{2} \bar{x}_{3}\right)\right\}
\end{aligned}
$$

Next, we will consider the BDD that represents the characteristic function for incompletely specified multiple-output function.

DEFINITION 2.4. The BDD_for_CF of a multiple-output function $F=\left(f_{1}, f_{2}, \ldots, f_{m}\right)$ represents the characteristic function $\chi$ of $F$, where the variable representing the output $y_{i}$ is in the below of the support variables for $f_{i}$. (We assume that the root node is in the top.)

Fig. 2.1 illustrates a BDD_for_CF of an incompletely specified multiple-output function, where solid lines denote the 1-edges, while dotted lines denote the 0 -edges. When the 1-edge of the node $y_{i}$ is connected to a constant 0 node, $f_{i}=0$ (Fig. 2.1(a)); when the 0 -edge of the node $y_{i}$ is connected to a constant 0 node, $f_{i}=1$ (Fig. 2.1(b)); and when both the 0-edge and 1-edge of the node $y_{i}$ are connected to the same non-constant 0 node, $f_{i}=d$ (don't care) (Fig. 2.1(c)). In the case of $f_{i}=d$, the node for $y_{i}$ is redundant, and it is deleted during the minimization of the BDD.

In the case of a BDD_for_CF representing a completely specified function, each path from the root node to the constant 1 node involves nodes for all the output variables $y_{i}$. Furthermore, one of the edges of the node for $y_{i}$ is connected to the constant 0 node. On the other hand, in the case of a BDD_for_CF representing an incompletely specified function, each path from the root node to the constant 1 node may not involve nodes for some variable $y_{i}$. For the path where the output variables $y_{i}$ is missing, $f_{i}$ is don't care.
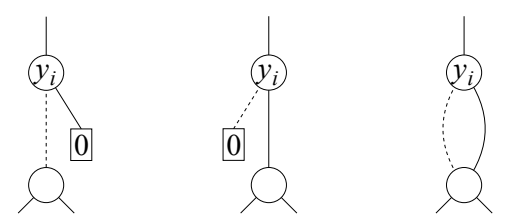
(a) $f_{i}=0$
(b) $f_{i}=1$
(c) $f_{i}=d$

Figure 2.1: BDD_for_CF representing an incompletely specified function.

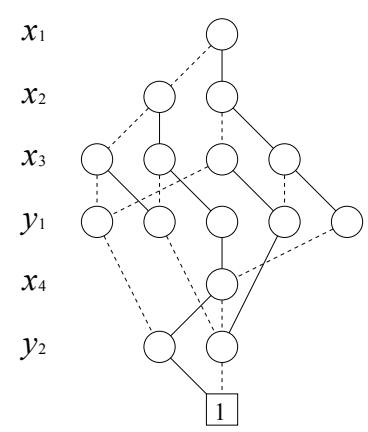

(a) BDD_for_CF when 0's are assigned to the all the don't cares.

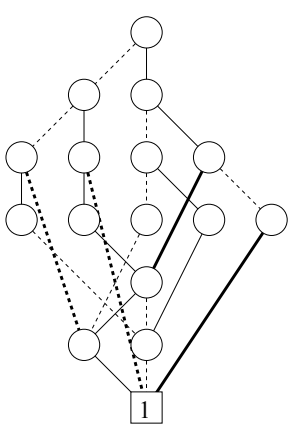

(b) BDD_for_CF for incompletely specified function.
Figure 2.2: BDD_for_CF representing multiple-output function.

EXAMPLE 2.2. Fig. 2.2 shows two BDD_for_CFs representing the function in Example 2.1. For simplicity, the constant 0 node and all the edges connecting to it are omitted. Fig. 2.2(a) shows the BDD_for_CF representing the completely specified function, where 0's are assigned to all the don't cares. Fig. 2.2(b) shows the BDD_for_CF representing the incompletely specified function. The solid and dotted bold edges denote that at least one node for an output variable is missing, and the output value is don't care. Note that in Fig. 2.2(a), all the output variables $\left\{y_{1}, y_{2}\right\}$ appear in each path from the root node to the constant 1 node. On the other hand, in Fig. 2.2(b), in the bold edges at least one output variable $y_{i}$ is missing, and the corresponding output $f_{i}$ is don't care.

\section{DECOMPOSITION AND BDD_FOR_CFS}

\subsection{Decomposition using BDD_for_CF}

By using a BDD_for_CF, we can decompose a multiple-output logic function efficiently [14]. When we decompose the function by using a BDD, the smaller the width of the BDD, the smaller the network becomes after decomposition. In the case of a incompletely specified function, we can often reduce the width of the BDD_for_CF by finding an appropriate assignment of constants to the don't cares. From here, we will consider a method to reduce the width of a BDD_for_CF representing an incompletely specified function.

DEFINITION 3.1. Let the height of the root node be the total number of variables, and let the height of the constant node be 0 . Let $\left(z_{n+m}, z_{n+m-1}, \ldots, z_{1}\right)$ be the ordering of the variables, where $z_{n+m}$ corresponds to the variable for the root node. The width of the BDD_for_CF at the height $k$ is the number of edges crossing the section of the BDD between variables $z_{k}$ and 
Table 3.1: Decomposition chart of an incompletely specified function.

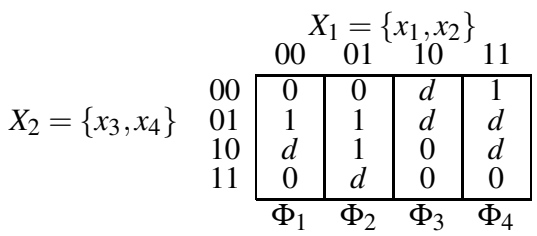

$z_{k+1}$, where the edges incident to the same node are counted as one, also the edges pointing the constant $O$ are not counted. The width of the BDD_for_CF at the height $O$ is defined as 1.

DEFINITION 3.2. Let $f(X)$ be a logic function, and $\left(X_{1}, X_{2}\right)$ be a partition of the input variables. Let $|X|$ be the number of elements in $X$. The decomposition chart for $f$ is a twodimensional matrix with $2^{\left|X_{1}\right|}$ columns and $2^{\left|X_{2}\right|}$ rows, where each column and row has a label of unique binary code, and each element corresponds the truth value of $f$. In the decomposition chart, the column multiplicity denoted by $\mu$ is the number of different column patterns ${ }^{1}$. The function represented by a column is a column function.

EXAMPLE 3.1. Table 3.1 shows a decomposition chart of a 4-input 1-output incompletely specified function. Since all the column patterns are different, the column multiplicity is $\mu=4$.

In a conventional functional decomposition using a BDD, the width of a BDD is equal to the column multiplicity $\mu$. Let $\left(X_{1}, X_{2}\right)$ be a partition of the input variables, then the nodes except for variables $X_{1}$ that are directly connected to the nodes for variables $X_{1}$ correspond to the column patterns in the decomposition chart. In a partition $\left(X_{1}, X_{2}\right)$, nodes representing column functions may have different heights. In a functional decomposition, such a relation is denoted by $f\left(X_{1}, X_{2}\right)=g\left(h\left(X_{1}\right), X_{2}\right)$. When $\left\lceil\log _{2} \mu\right\rceil<\left|X_{1}\right|$, the function $f$ can be decomposed into two networks: The first one realizes $h\left(X_{1}\right)$, and the second one realizes $g\left(h, X_{2}\right)$. The functional decomposition is effective when the number of inputs for $g$ is smaller than that of $f$.

DEFINITION 3.3. Two incompletely specified functions $f$ and $g$ are compatible, denoted by $f \sim g$, iff $f_{-} \cdot g_{-1}=0$ and $f_{-1}$. $g_{-} 0=0$.

LEMMA 3.1. Let $\chi_{a}$ and $\chi_{b}$ be the characteristic functions of two incompletely specified functions. If $\chi_{a} \sim \chi_{b}$ and $\chi_{c}=\chi_{a} \chi_{b}$, then $\chi_{c} \sim \chi_{a}$ and $\chi_{c} \sim \chi_{b}$.

EXAMPLE 3.2. In the decomposition chart of Table 3.1, for pairs of column functions $\left\{\Phi_{1}, \Phi_{2}\right\},\left\{\Phi_{1}, \Phi_{3}\right\}$, and $\left\{\Phi_{3}, \Phi_{4}\right\}$, the functions are compatible. Make the logical product of columns $\Phi_{1}$ and $\Phi_{2}$, and replace them with $\Phi_{1}^{*}$ and $\Phi_{2}^{*}$, respectively. Where, $\Phi_{1}^{*}$ and $\Phi_{2}^{*}$ show that the functions obtained by assigning constants to don't cares. Also, make the logical product of columns $\Phi_{3}$ and $\Phi_{4}$, and replace them with $\Phi_{3}^{*}$ and $\Phi_{4}^{*}$, respectively. Then, we have the decomposition chart in Table 3.2, where $\mu=2$.

The following theorem is similar to, but different from well known theorem on conventional functional decomposition using BDDs [8, 13]. It is an extension of [14] into incompletely specified functions.

${ }^{1}$ In the case of BDD_for_CF, we do not count the columns that consist of all zeros.
Table 3.2: Reduction of column multiplicity.

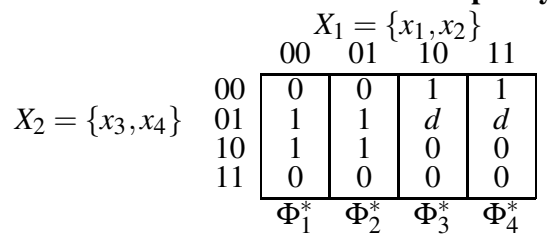

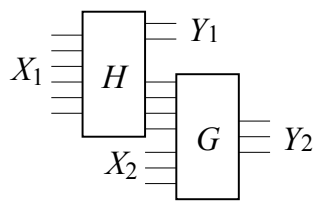

Figure 3.1: Decomposition of multiple-output function.

THEOREM 3.1. Let $\left(X_{1}, Y_{1}, X_{2}, Y_{2}\right)$ be the variable ordering of the BDD_for_CF that represents the incompletely specified function, where $X_{1}$ and $X_{2}$ denote the disjoint ordered sets of input variables, and $Y_{1}$ and $Y_{2}$ denote the disjoint ordered sets of output variables. Let $n_{2}$ be the number of variables in $X_{2}$, and $m_{2}$ be the number of variables in $Y_{2}$. Let $W$ be the width of the BDD for $C F$ at height $n_{2}+m_{2}$. When counting the width $W$, ignore the edges that connect the nodes of output variables and the constant 0 . Suppose that the multiple-output function is realized by the network shown in Fig. 3.1. Then, the necessary and sufficient number of connections between two blocks $H$ and $G$ is $\left\lceil\log _{2} W\right\rceil$.

\subsection{Algorithm to Reduce the Width of a BDD_for_CF}

Various methods exist to reduce the number of nodes in BDDs representing incompletely specified functions $[3,6,18,19,20]$. In the method [19], for each node, two children are merged when the functions represented by them are compatible. For example, when two children $f$ and $g$ in Fig. 3.2(a) are compatible, the BDD is simplified as shown in Fig. 3.2(b). By doing this operation repeatedly, we can reduce the number of nodes in the BDD. The following algorithm is used in our experiment, which is a simplified version of [19]. Note that our data structure is a BDD_for_CF instead of an SBDD.

ALGORITHM 3.1. From the root node of the BDD, do the following operations recursively.

1. If the function represented by node $v$ has no don't care, then terminate.

2. For $v$, check if two children $v_{0}$ and $v_{1}$ are compatible. Let the functions represented by $v_{0}$ and $v_{1}$ be $\chi_{0}$ and $\chi_{1}$, respectively.

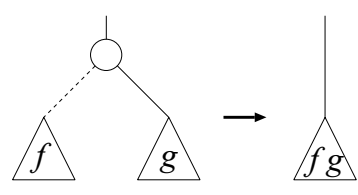

(a)

(b)

Figure 3.2: Simplification method in [19]. 


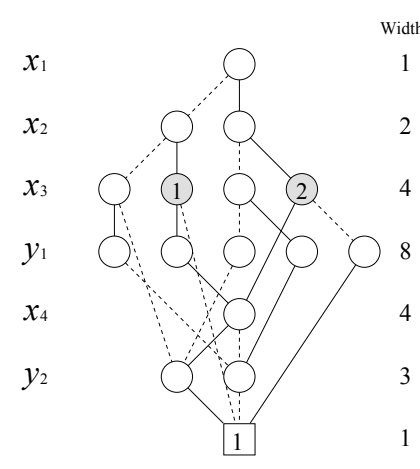

(a) Before application.

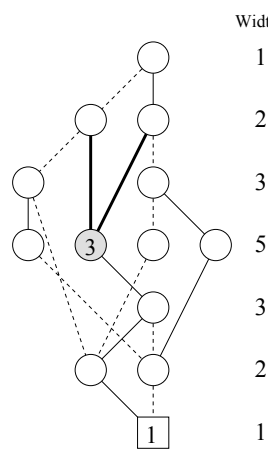

(b) After application.

Figure 3.3: BDD_for_CF before and after application of Algorithm 3.1.

- If they are incompatible, then apply this algorithm to $v_{0}$ and $v_{1}$

- If they are compatible, then replace $v_{0}$ and $v_{1}$ with $v_{\text {new }}$, where $v_{\text {new }}$ represents $\chi_{\text {new }}=\chi_{0} \cdot \chi_{1}$.

EXAMPLE 3.3. Fig. 3.3(a) and (b) show the BDD_for_CFs before and after application of Algorithm 3.1, respectively. When there is only one edge coming down from a node, it denotes two edges that coincide. In Fig. 3.3(a), nodes 1 and 2 have compatible two children. For this function, the node replaced for node 1 , and the node replaced for nodes 2 are the same. So, in Fig. 3.3(b), two nodes 1 and 2 are replaced with node 3. In the figures, the rightmost columns headed with "Width" denote the widths of the BDDs for each height. Note that the maximum width is reduced from 8 to 5 , and the number of non-terminal nodes is reduced from 15 to 12 .

The method in [19] is effective for local reduction of the number of nodes. However, since it only considers the compatibility of two children for each node at one time, it is not so effective to reduce the width of the BDD. Thus, in our method, we check the compatibility of column functions in each height, and perform the minimal clique cover of the functions to reduce the width of the BDD.

DEFINITION 3.4. In a compatibility graph, each node corresponds to a function, and an edge exists between nodes if the corresponding functions are compatible.

In the functional decomposition, check the compatibility of the column functions, and construct the compatibility graph. Then, minimize the column multiplicity $\mu$ by finding the minimum clique cover [20,3]. Since this problem is NP-hard [5], we use the following heuristic method.

Algorithm 3.2. (Heuristic Minimal Clique Cover) Let $S_{a}$ be the set of all the nodes in the compatibility graph. Let $C$ be the set of subset of $S_{a}$. From $S_{a}$, delete isolated nodes, and put them into $C$. While $S_{a} \neq \phi$, iterate the following operations:

1. Let $v_{i}$ be the node that has the minimum number of edges in $S_{a}$. Let $S_{i} \leftarrow\left\{v_{i}\right\}$. Let $S_{b}$ be the set of nodes in $S_{a}$ that are connecting to $v_{i}$.

2. While $S_{b} \neq \phi$, iterate the following operations:

(a) Let $v_{j}$ be the node that has the minimum edges in $S_{b}$. Let $S_{i} \leftarrow S_{i} \cup\left\{v_{j}\right\} . S_{b} \leftarrow S_{b}-\left\{v_{j}\right\}$.

(b) From $S_{b}$, delete the nodes that are not connected to $v_{j}$.

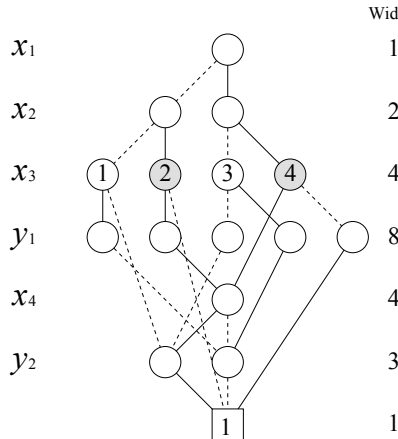

(a)

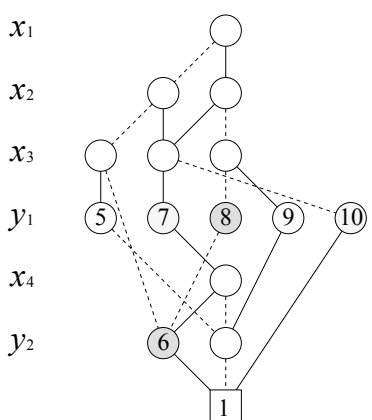

(c)

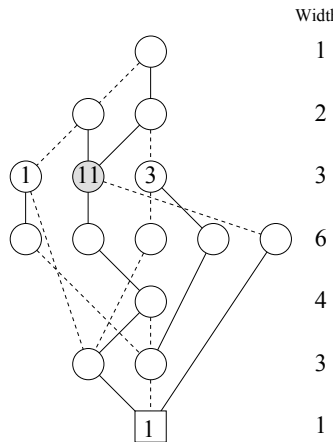

(b)

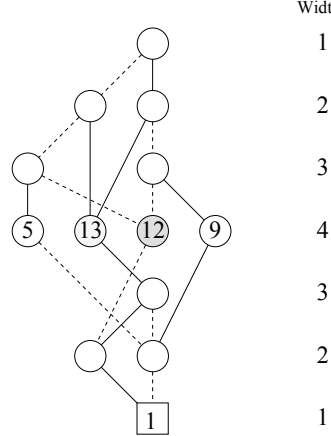

(d)
Figure 3.4: Reduction of width of BDD_for_CF using Algorithm 3.3.

3. $C \leftarrow \mathcal{C} \cup\left\{S_{i}\right\}, S_{a} \leftarrow S_{a}-S_{i}$.

ALGORITHM 3.3. (Reduction of Widths of a BDD_for_CF) Let the height of the root node be t, and let the height of the constant nodes be 0 . From the height $t-1$ to 1 , iterate the following operations:

1. For each height, construct the set of all the column functions, and construct the compatibility graph.

2. Find the minimum clique cover for the nodes by Algorithm 3.2 .

3. For the functions that corresponds the nodes of each clique, assign constants to the don't cares to make a function that is compatible to all the function.

4. For each column function, replace it with the function produced in step 3, and re-construct the BDD with a smaller width.

EXAMPLE 3.4. Fig. 3.4 shows the BDD_for_CF after applying Algorithm 3.3 to one in Fig. 2.2. At the height of $x_{3}$, nodes 2 and 4 are compatible in Fig. 3.4(a). So, these nodes are merged into node 11 in Fig. 3.4(b). Next, at the height of $y_{1}$, the compatibility graph is shown in Fig. 3.5. Note that in Fig. 3.4(c), nodes 6 and 8 are replaced by node 12 , and nodes 7 and 10 are replaced by node 13. The resulting BDD is shown in Fig. 3.4(d). By comparing Figs. 3.4(a) and (d), we can see that the maximum width is reduced from 8 to 4 , and the number of non-terminal nodes is reduced from 15 to 12 .

\section{EXPERIMENTAL RESULTS}

\subsection{Benchmark Functions}

To evaluate the performance of Algorithm 3.3, we generated the following incompletely specified functions $[16,15]$ : 


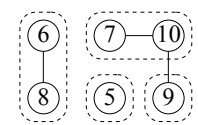

Figure 3.5: Compatibility graph.

\section{Residue Number to Binary Number Converters:}

- 5-7-11-13 RNS (14-input 13-output, DC 69.5\%: 4-digit RNS number [7], where the moduli are 5, 7, 9, and 11)

- 7-11-13-17 RNS (16-input 15-output, DC 74.0\%)

- 11-13-15-17 RNS (17-input 16-output, DC 72.2\%)

\section{k-nary to Binary Converters:}

- 4-digit 11-nary to binary (16-input 14-output, DC 77.7\%)

- 4-digit 13-nary to binary (16-input 15-output, DC 56.4\%)

- 5-digit decimal to binary (20-input 17-output, DC $90.5 \%$ )

- 6-digit 5-nary to binary (18-input 14-output, DC 94.0\%)

- 6-digit 6-nary to binary (18-input 16-output, DC $82.2 \%$ )

- 6-digit 7-nary to binary (18-input 17 -output, DC $55.1 \%$ )

- 10-digit ternary to binary (20-input 16-output, DC 94.4\%)

\section{Decimal Adders and a Multiplier:}

- 3-digit decimal adder (24-input 16-output, DC 94.0\%)

- 4-digit decimal adder (32-input 20-output, DC 97.7\%)

- 2-digit decimal multiplier (16-input 16-output, DC $84.7 \%$ )

EXAMPLE 4.1. Consider the 10-digit ternary to binary converters. Binary-coded-ternary is used to represent a ternary digit: 0 is represented by (00); 1 is represented by (01); and 2 is represented by (10). (11) is an undefined input, and the corresponding outputs are don't cares. Thus, $\left(\frac{3}{4}\right)^{10}=0.0563$ of the inputs are specified, but $1-\left(\frac{3}{4}\right)^{10}=0.9436$ are don't cares.

\subsection{Reduction of BDD Width}

We applied Algorithms 3.1 and 3.3 to each of the incompletely specified function presented in Section 4.1, and reduced the widths of the BDD_for_CF. Before applying Algorithms, we optimize the variable order for the BDD_for_CF by sifting algorithm [12], where the sum of widths is used as the cost function.

When all the outputs of the function are represented by a single BDD_for_CF, the circuits are too large to implement. So, we partition the outputs into two sets, and represent each of them by a BDD_for_CF separately. Table 4.1 shows maximum widths of BDD_for_CF when the multiple-output function $F=$ $\left(f_{1}, \ldots, f_{m}\right)$ is partitioned into two: $F_{1}=\left(f_{1}, \ldots, f_{\lceil m / 2\rceil}\right)$ and $F_{2}=\left(f_{\lceil m / 2\rceil+1}, \ldots, f_{m}\right)$. The upper numbers denote the results for $F_{1}$, and the lower numbers denote the results for $F_{2}$. In the tables, the column headed by $D C=0$ denotes the case where constant 0 's are assigned to all the don't cares; $D C=1$ denotes the case where constant 1 's are assigned to all the don't cares; ISF denotes the case where incompletely specified functions (ternary functions) are represented; Alg3.1 denotes the case where Algorithm 3.1 is applied; and Alg3.3 denotes the case where Algorithm 3.3 is applied. Reduction ratio is normalized to 1.00 for the case of $D C=0$.

By partitioning the outputs into two sets, we could drastically reduce the sizes of the BDDs for all the functions. For some function, the maximum width of the BDD became $1 / 2000$, and the total number of nodes became $1 / 70$. With bi-partitions, we can implement the circuits with reasonable sizes.

Table 4.1 shows that Algorithm 3.3 produced BDDs with smaller widths than Algorithm 3.1, especially for $F_{2}$, the outputs for less
Table 4.1: Maximum width of BDD_for_CFs.

\begin{tabular}{|l|r|r|r|r|r|}
\hline \multicolumn{1}{|c|}{ Name } & DC=0 & DC=1 & ISF & Alg3.1 & Alg3.3 \\
\hline 5-7-11-13 RNS & 503 & 503 & 503 & 502 & 416 \\
& 461 & 461 & 461 & 460 & 363 \\
\hline 7-11-13-17 RNS & 471 & 471 & 471 & 470 & 337 \\
& 1089 & 1089 & 1089 & 1088 & 896 \\
\hline 11-13-15-17 RNS & 1574 & 1574 & 1574 & 1573 & 1573 \\
& 2253 & 2253 & 2254 & 2253 & 1229 \\
\hline 4-digit 11-nary to binary & 117 & 117 & 129 & 123 & 117 \\
& 257 & 257 & 264 & 264 & 128 \\
\hline 4-digit 13-nary to binary & 226 & 226 & 236 & 232 & 225 \\
& 257 & 257 & 264 & 264 & 128 \\
\hline 5-digit decimal to binary & 393 & 393 & 393 & 392 & 391 \\
& 257 & 257 & 78 & 76 & 64 \\
\hline 6-digit 5-nary to binary & 134 & 134 & 155 & 148 & 139 \\
& 257 & 257 & 260 & 260 & 128 \\
\hline 6-digit 6-nary to binary & 185 & 185 & 189 & 188 & 184 \\
& 257 & 257 & 89 & 64 & 32 \\
\hline 6-digit 7-nary to binary & 464 & 464 & 485 & 482 & 472 \\
& 513 & 513 & 516 & 516 & 256 \\
\hline 10-digit ternary to binary & 265 & 265 & 305 & 304 & 294 \\
& 513 & 513 & 514 & 514 & 256 \\
\hline 3-digit decimal adder & 29 & 25 & 15 & 14 & 10 \\
& 200 & 101 & 14 & 13 & 10 \\
\hline 4-digit decimal adder & 85 & 73 & 15 & 14 & 10 \\
& 1400 & 649 & 14 & 13 & 10 \\
\hline 2-digit decimal multiplier & 945 & 946 & 955 & 954 & 945 \\
& 767 & 769 & 327 & 269 & 224 \\
\hline Reduction ratio & 1.000 & 0.950 & 0.825 & 0.811 & 0.636 \\
\hline
\end{tabular}

Table 4.2: Reduction of LUT Cascades by using don't cares.

\begin{tabular}{|c|c|c|c|c|c|c|}
\hline \multirow[t]{2}{*}{ Name } & \multicolumn{3}{|c|}{$\mathrm{DC}=0$} & \multicolumn{3}{|c|}{ Alg3.3 } \\
\hline & \#Cells & \#LUTs & \#Cas & \#Cells & & \#Cas \\
\hline 5-7-11-13 RNS & 6 & 35 & 3 & 4 & 29 & 2 \\
\hline 7-11-13-17 RNS & 9 & 53 & 3 & 8 & 52 & \\
\hline 11-13-15-17 RNS & 19 & 118 & 5 & 18 & 108 & \\
\hline 4-digit 11 -nary to binary & 4 & 29 & 2 & 4 & 28 & \\
\hline 4-digit 13 -nary to binary & 4 & 31 & 2 & 4 & 30 & \\
\hline 5-digit decimal to binary & 8 & 50 & 3 & 7 & 48 & \\
\hline 6-digit 5-nary to binary & 6 & 44 & 2 & 5 & 36 & \\
\hline 6-digit 6-nary to binary & 5 & 38 & 2 & 5 & 29 & \\
\hline 6-digit 7-nary to binary & 10 & 68 & 3 & 9 & 58 & 3 \\
\hline 10-digit ternary to binary & 9 & 59 & 3 & 6 & 48 & 2 \\
\hline 3-digit decimal adder & 7 & 37 & 2 & 3 & 17 & 1 \\
\hline 4-digit decimal adder & 10 & 64 & 2 & 4 & 24 & \\
\hline 2-digit decimal multiplier & 8 & 51 & 3 & 7 & 48 & 3 \\
\hline Total & 105 & 677 & 35 & 84 & 555 & 30 \\
\hline Ratio & 1.00 & 1.00 & 1.00 & 0.80 & 0.82 & 0.86 \\
\hline
\end{tabular}

significant bits. Algorithm 3.1 checks only the compatibility of two children for each node, and reduces the number of nodes locally. On the other hand, Algorithm 3.3 checks compatibilities among functions for each height of a BDD, and so reduces the width more effectively. Let $w$ be the width of the BDD, then the algorithm checks $\left(w^{2}-w\right) / 2$ compatibilities. Also, it performs minimal clique cover, so it is more time-consuming than Algorithm 3.1.

We used gec for Red Hat Linux version 2.4 on a PC using Pentium $4(2.8 \mathrm{GHz})$ with $4 \mathrm{G}$ Byte memory. The longest CPU times were as follows: When all the outputs were represented by a single BDD_for_CF: $135 \mathrm{sec}$ (7-11-13-17 RNS) to generate BDD_for_CF. $783 \mathrm{sec}$ (10-digit ternary to binary) to reduce the width of the BDD. When the outputs are bi-partitioned: $26 \mathrm{sec}$ (11-13-15-17 RNS) to generate a pair of BDD_for_CFs. $11 \mathrm{sec}$ (11-13-15-17 RNS) to reduce the widths of BDD_for_CFs.

\subsection{Logic Synthesis of LUT Cascades}

In the practical applications, when the width of the BDD is slightly larger than $2^{k}$, by properly assigning the constants to don't cares, we can often reduce the width of the BDD, and re- 


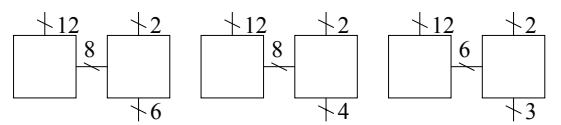

(a) When 0's were assigned to all the don't cares.

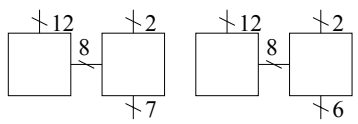

(b) When Algorithm 3.3 was used to assign don't cares.

Figure 4.1: 5-7-11-13 RNS to binary number converters.

duces the number of interconnections between two blocks $H$ and $G$ in Fig. 3.1.

To see the usefulness of Algorithm 3.3, we designed benchmark functions in Section 4.1 by LUT cascades [14] . Table 4.2 shows the sizes of LUT cascades. For all benchmark functions except for 11-13-15-17 RNS, we assume that each cell has at most 12 inputs and at most 8 outputs [11]. For 11-13-15-17 RNS, we used cells with at most 12 inputs and at most 9 outputs, since it required 9-output cells. In the table, \#Cells denote the number of cells in the cascade; \#LUTs denotes total number of LUT outputs; and \#Cas denotes the number of cascades.

For example, consider 5-7-11-13 RNS. In this function, 69.5\% of the input combinations are don't cares. Fig. 4.1(a) shows the case where constant 0 's were assigned to all the don't cares, while Fig. 4.1(b) shows the case where Algorithm 3.3 was used to assign don't cares. Algorithm 3.3 produced smaller cascades: On the average, the total numbers of cells is reduced by by $20 \%$, the total number of cell outputs is reduced by $18 \%$, and the total numbers of cascades is reduced by $14 \%$.

We also designed cascades by using Algorithm 3.1. In this case, the reduction were, $15 \%, 14 \%$, and $11 \%$, respectively. So, it was not so effective as Algorithm 3.3.

\section{CONCLUDING REMARKS}

In this paper, we first showed a new method to represent an incompletely specified multiple-output function by a BDD_for_CF. Then, we presented a method to reduce the width of the BDD. Finally, we applied this method to radix converters, adders and a multiplier. When all the outputs were represented by a single BDD_for_CF, we could not reduce the width of the BDD even if we use the don't cares. However, when the outputs were partitioned into two sets, and each set was represented by a BDD_for_CF, we could reduce the width of the BDD by using don't cares. We also applied this method to design LUT cascades. By using the method, we could reduce the numbers of cells in cascades, on the average, by $20 \%$.

The technique is promising for other BDD-based synthesis areas, e.g. BDD-based state-space exploration, where the BDD representation of the state set can be simplified using reached states as don't cares.

\section{Acknowledgments}

This research is supported in part by Grant in Aid for Scientific Research of MEXT, and Grant of Kitakyushu Area Innovative Cluster Project.

\section{REFERENCES}

[1] R. L. Ashenhurst, "The decomposition of switching functions," International Symposium on the Theory of Switching, pp. 74-116, April 1957.
[2] P. Ashar and S. Malik, "Fast functional simulation using branching programs," Inter. Conf. on CAD, pp. 408-412, Nov. 1995.

[3] S. Chang, D. Cheng, and M. Marek-Sadowska, "Minimizing ROBDD size of incompletely specified multiple output functions," In European Design \& Test Conf., pp. 620-624, 1994.

[4] K. Cho and R. E. Bryant, "Test pattern generation for sequential MOS circuits by symbolic fault simulation," Design Automation Conference, pp. 418-423, June 1989.

[5] M. R. Garey and D. S. Johnson, Computers and Intractability, Freeman, San Francisco, 1979.

[6] Y. Hong, P. Beerel, J. Burch, and K. McMillan, "Safe BDD minimization using don't cares," Design Automation Conference, pp. 208-213, 1997.

[7] I. Koren ,Computer Arithmetic Algorithms (2nd Edition), A. K. Peters, Natick, MA, 2002.

[8] Y-T. Lai, M. Pedram and S. B. K. Vrudhula, "BDD based decomposition of logic functions with application to FPGA synthesis", Design Automation Conference, 1993.

[9] S. Minato, N. Ishiura, and S. Yajima, "Shared binary decision diagram with attributed edges for efficient Boolean function manipulation," Design Automation Conference, pp. 52-57, June 1990.

[10] A. Mishchenko and T. Sasao, "Encoding of Boolean functions and its application to LUT cascade synthesis," International Workshop on Logic and Synthesis 2002, pp. 115-120, New Orleans, Louisiana, June 4-7, 2002.

[11] K. Nakamura, and T. Sasao, et.al "Programmable logic device with an 8-stage cascade of 64K-bit asynchronous SRAMs," Cool Chips VIII, IEEE Symposium on Low-Power and High-Speed Chips, April 20-22, 2005 , Yokohama, Japan (to be published).

[12] R. Rudell, "Dynamic variable ordering for ordered binary decision diagrams," ICCAD-93, pp. 42-47, 1993.

[13] T. Sasao, "FPGA design by generalized functional decomposition," In Logic Synthesis and Optimization, Kluwer Academic Publisher, pages 233-258, 1993.

[14] T. Sasao and M. Matsuura, "A method to decompose multiple-output logic functions," Design Automation Conference, pp. 428-433, San Diego, June 2-6, 2004.

[15] T. Sasao, "Radix converters: Complexity and implementation by LUT cascades," International Symposium on Multiple-Valued Logic, May 2005 (to be published).

[16] Available at http://www.lsi-cad.com/dac2005

[17] M. Sauerhoff and I. Wegener, "On the complexity of minimizing the OBDD size for incompletely specified functions," IEEE Transactions on TCAD, Vol. 15, No. 11, pp. 1435-1437, 1996.

[18] C. Scholl, "Multi-output functional decomposition with exploitation of don't cares," In Design Automation and Test Europe, pp. 743-748, Feb. 1998.

[19] T. R. Shiple, R. Hojati, A. L. Sangiovanni-Vincentelli, and R. K. Brayton, "Heuristic minimization of BDDs using don't cares," Design Automation Conference, pp. 225-231, 1994.

[20] W. Wan and M. A. Perkowski, "A new approach to the decomposition of incompletely specified functions based on graph-coloring and local transformations and its application to FPGA mapping," IEEE EURO-DAC'92, pp. 230-235, Hamburg, Sept. 7-10, 1992. 